\title{
DISCOVERY OF A LOW-MASS COMPANION TO THE SOLAR-TYPE STAR TYC 2534-698-1
}

\author{
Stephen R. Kane ${ }^{1}$, Suvrath Mahadevan ${ }^{2}$, William D. Cochran ${ }^{3}$, Rachel A. Street ${ }^{4}$, Thirupathi Sivarani ${ }^{2}$, Gregory $^{5}$ \\ W. HenRY ${ }^{5}$, AND Michael H. Williamson ${ }^{5}$ \\ ${ }^{1}$ NASA Exoplanet Science Institute, Caltech, MS 100-22, 770 South Wilson Avenue, Pasadena, CA 91125, USA; skane@ipac.caltech.edu \\ ${ }^{2}$ Department of Astronomy, University of Florida, 211 Bryant Space Science Center, Gainesville, FL 32611-2055, USA \\ ${ }^{3}$ Department of Astronomy, University of Texas, Austin, TX 78712, USA \\ ${ }^{4}$ Las Cumbres Observatory Global Telescope, Goleta, CA 93117, USA \\ ${ }^{5}$ Center of Excellence in Information System, Tennessee State University, 3500 John A. Merritt Blvd., Box 9501, Nashville, TN 37209, USA \\ Received 2008 September 23; accepted 2008 November 10; published 2009 February 18
}

\begin{abstract}
Brown dwarfs and low-mass stellar companions are interesting objects to study since they occupy the mass region between deuterium and hydrogen burning. We report here the serendipitous discovery of a low-mass companion in an eccentric orbit around a solar-type main-sequence star. The stellar primary, TYC 2534-698-1, is a G2V star that was monitored both spectroscopically and photometrically over the course of several months. Radial velocity observations indicate a minimum mass of $0.037 M_{\odot}$ and an orbital period of $\sim 103$ days for the companion. Photometry outside of the transit window shows the star to be stable to within $\sim 6$ millimags. The semimajor axis of the orbit places the companion in the "brown dwarf desert" and we discuss potential follow-up observations that could constrain the mass of the companion.
\end{abstract}

Key words: planetary systems - stars: low-mass, brown dwarfs

\section{INTRODUCTION}

Brown dwarfs in short and intermediate periods $(<5 \mathrm{AU})$ are relatively rare compared to their occurrence at wider separations. Metchev \& Hillenbrand (2008) show that the frequency of brown dwarfs at larger separations (29-1590 AU) is $~ 3.2 \%$, much larger than the $\sim 0.5 \%$ observed for separations less than 3 AU (Marcy \& Butler 2000). This "brown dwarf desert" in radial velocity observations is highly significant and not easy to explain. Confirmation of radial velocity detected brown dwarf candidates is also challenging since the observations only yield a minimum mass and radial velocity alone cannot break the degeneracy between companion mass and inclination.

For some of the brighter stars hosting brown dwarf candidates, the Hippparcos measurements (Perryman et al. 1997) (coupled with the radial velocity data) can be used to estimate the mass of the companion. Such techniques have been demonstrated by Zucker \& Mazeh (2000), Reffert \& Quirrenbach (2006), and Kürster et al. (2008). Eclipsing binary systems, and close binaries where both spectra can be observed, offer the prospect of the dynamical measurement of the mass of the two companions, and such systems have been used to determine accurate brown dwarf and low-mass star masses by Zapatero Osorio et al. (2004) and Stassun et al. (2006). Ireland et al. (2008) have measured the dynamical mass for GJ 802 b using aperture masking and interferometry.

Another way to confirm the mass of the companion unambiguously is to detect a transit. Coupled with radial velocity data this constrains the inclination angle and therefore the mass of the companion. The challenge with attempting this method on brown dwarf candidates discovered using the radial velocity technique is that the periods are typically 100-1000 days, making the transit probability very small. Another approach is to follow-up up detections from transit surveys with radial velocity observations to determine the mass of the candidate. This method has recently been employed by Deleuil et al. (2008) in the discovery of the transiting brown dwarf ( $\sim 22$ Jupiter mass) companion to the F3V star CoRoT-Exo-3. While this is frequently done for the most promising candidates, the large amount of time required for precision radial velocity observations to confirm a planet leads to many candidates also being rejected before radial velocity follow-up. We selected five candidate transiting objects from the SuperWASP fields between $06 \mathrm{hr}$ and $16 \mathrm{hr}$ (Kane et al. 2008) for radial velocity follow-up. Our motivation was to confirm either a brown dwarf or a lowmass stellar companion. No confirmed transiting brown dwarf around relatively bright stars $(V<12)$ has been discovered yet, although the planet XO-3b (Johns-Krull et al. 2008) may be massive enough for deuterium burning. Transiting low-mass stars are also interesting in their own right since an estimate of both mass and radii helps to constrain the equation of state used in current models (e.g., Chabrier \& Baraffe 2000). In this paper, we present our radial velocity and photometric observations for one of our candidates, TYC 2534-698-1, which has a Tycho-2 $V$ magnitude of 10.8. For this object the SuperWASP data show a transit depth of 17.9 millimags and a periodicity of $\sim 2.67$ days. We find no radial velocity variability at this period, but our radial velocities show the existence of a $0.037 M_{\odot}$ low-mass companion (minimum mass) at a 103.69 day orbit.

\section{OBSERVATIONS}

In this section, we discuss the acquisition of the radial velocity and photometric data.

\subsection{Radial Velocity}

Radial velocity observation of TYC 2534-698-1 were obtained using the High Resolution Spectrograph (HRS, Tull 1998) on the Hobby-Eberly Telescope (HET) in queue scheduled mode. The observations used a 2 arcsec fiber, resulting in a spectral resolution of $R=60,000$. Thirteen observations were acquired with the HRS with an iodine cell placed in the stellar beam path. One stellar template exposure was acquired without the iodine cell in order to facilitate the extraction of precise radial velocities from the combined star and iodine spectra. On sky integration time ranged from $10 \mathrm{~min}$ to $15 \mathrm{~min}$. The queue 

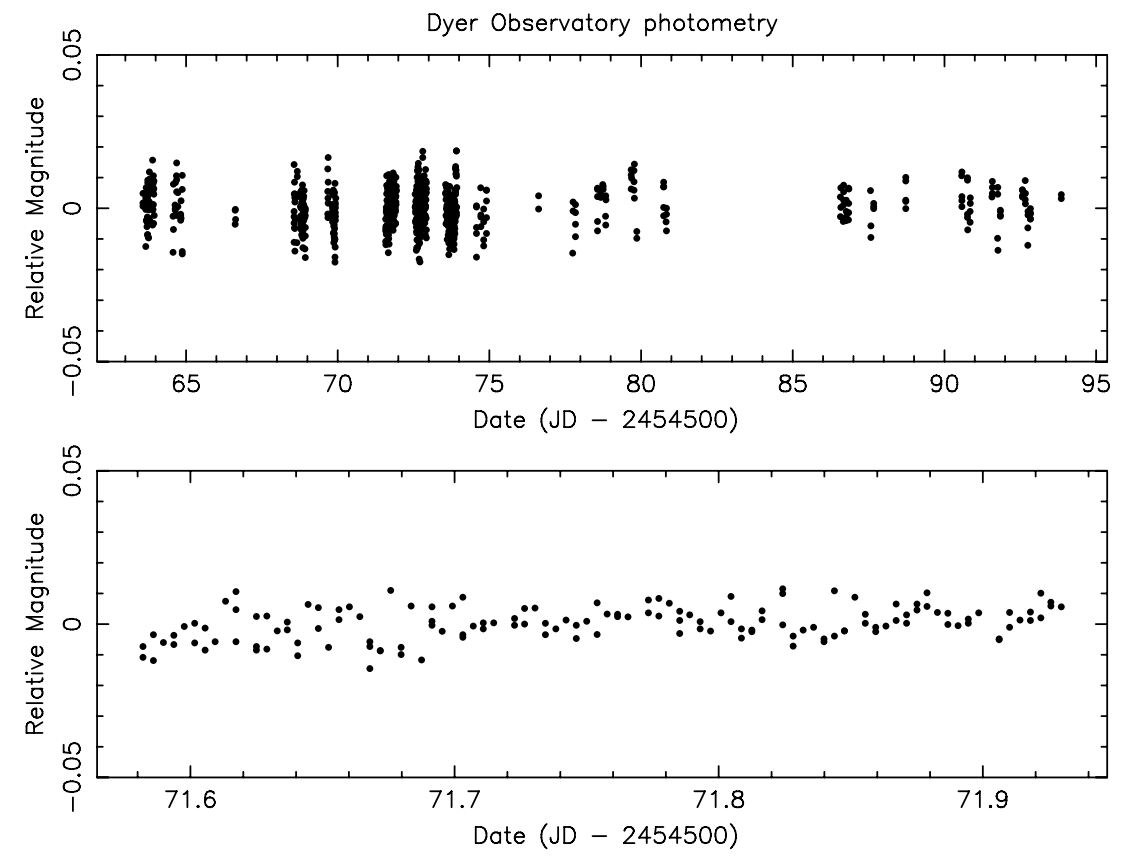

Figure 1. Photometry of TYC 2534-698-1 obtained using an automated 14 inch imaging telescope at Dyer Observatory.

scheduled nature of the HET allows great flexibility in the observations. The observations were placed on hold many times while the data were reduced in order to determine the best period. This made it possible to request observations that would be most useful in constraining the period of the companion. Our initial cadence was designed to detect the signature of the claimed SuperWASP periodicity of $P \sim 2.67$ days. The highresolution spectroscopic data obtained with the iodine cell were reduced by the method described in Cochran et al. (2004) using the stellar template and high-resolution iodine spectrum to simultaneously model instrumental and Doppler velocity shifts as well as variations in the point-spread function of the spectrograph. This technique allows the instrument drift to be calibrated out, and radial velocity precision of better than $2-3 \mathrm{~ms}^{-1}$ has been demonstrated on bright stars. Good velocity precision can only be obtained in the spectral region where iodine has sharp absorption lines, and therefore only the 5000-6200 $\AA$ region is used to derive velocities. Table 1 lists the radial velocities obtained for TYC 2534-698-1. The dominant source of velocity error is the photon noise limited radial velocity uncertainty, mainly due to the target being faint and the exposure time being short. The error bars stated are determined from the scatter in the velocities obtained from $2 \AA$ chunks of the spectrum.

The radial velocities are not consistent with the expected period from the SuperWASP data, but show the presence of a longer period companion. We discuss the fits to the radial velocity data in Section 3.2.

\subsection{Photometry}

Our target was monitored photometrically for over 30 days with three separate telescopes. The objectives of these observations were twofold: Firstly to provide confirmation of the transit of the low-mass companion, and secondly to determine the photometric stability of the target. The telescopes used were an automated 14 inch imaging telescope mounted at Dyer Observatory, Tennessee, a 16 inch telescope located at Las Cumbres Observatory (LCO) in California, and the Tenagra II automated 32 inch telescope located in Arizona.
Table 1

HET Radial Velocities for TYC 2534-698-1

\begin{tabular}{lcc}
\hline \hline Julian Date & Velocity $\left(\mathrm{ms}^{-1}\right)$ & Velocity Error $\left(\mathrm{ms}^{-1}\right)$ \\
\hline 2454515.809038 & -2449.63 & 29.33 \\
2454516.802895 & -2381.23 & 21.02 \\
2454518.789397 & -1955.85 & 21.47 \\
2454523.797391 & -705.29 & 22.06 \\
2454550.939348 & 227.73 & 22.87 \\
2454551.938358 & 185.48 & 23.33 \\
2454552.929063 & 149.75 & 23.74 \\
2454553.707716 & 133.50 & 23.35 \\
2454554.699371 & 99.05 & 21.60 \\
2454562.909758 & -271.73 & 24.55 \\
2454575.875428 & -853.48 & 23.58 \\
2454611.773732 & -2958.58 & 25.17 \\
2454627.726562 & -663.11 & 28.62 \\
\hline
\end{tabular}

As radial velocity data were obtained, the predicted time of transit was calculated based on the current best fit to the data (see Section 3.4). Since the best-fit orbital period shifted substantially from the original SuperWASP period, the time of predicted transit changed several times over the course of the HET observations. As such, photometry was obtained over a series of nights as the model parameters for the orbit were updated.

Shown in Figure 1 are the photometric measurements obtained using the Dyer Observatory 14 inch telescope coupled to an SBIG ST-1001E CCD camera. The top panel presents the photometry acquired over the course of $\sim 30$ days, and the bottom panel presents the photometry from a single night. The relative magnitudes were produced by comparing the brightness of TYC 2534-698-1 to the mean brightness of six constant stars in the same field. The star appears to be quite stable, with a standard deviation of 6.2 millimags. No evidence of a transit was detected in this dataset.

Figure 2 shows the photometry obtained with the LCO 16 inch telescope covering $\sim 10$ days. Once again, the star is very stable on the dates observed, with a standard deviation of 

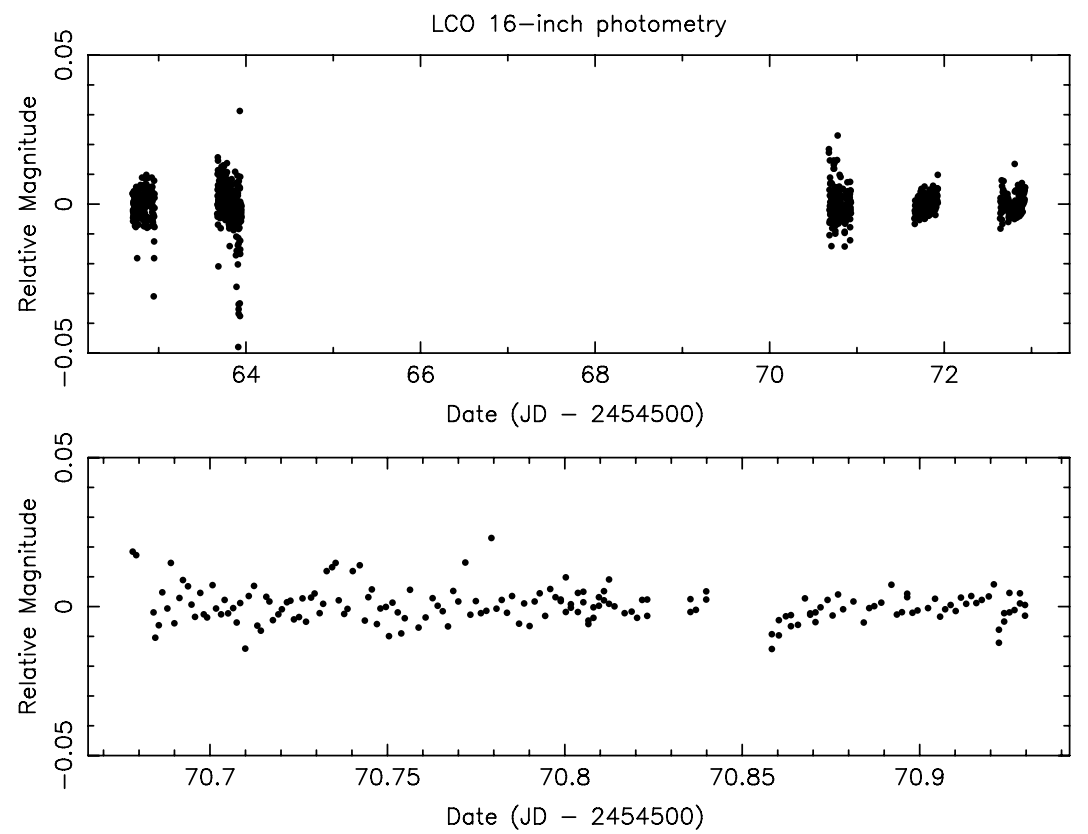

Figure 2. Photometry of TYC 2534-698-1 obtained using a 16 inch telescope mounted at Las Cumbres Observatory.
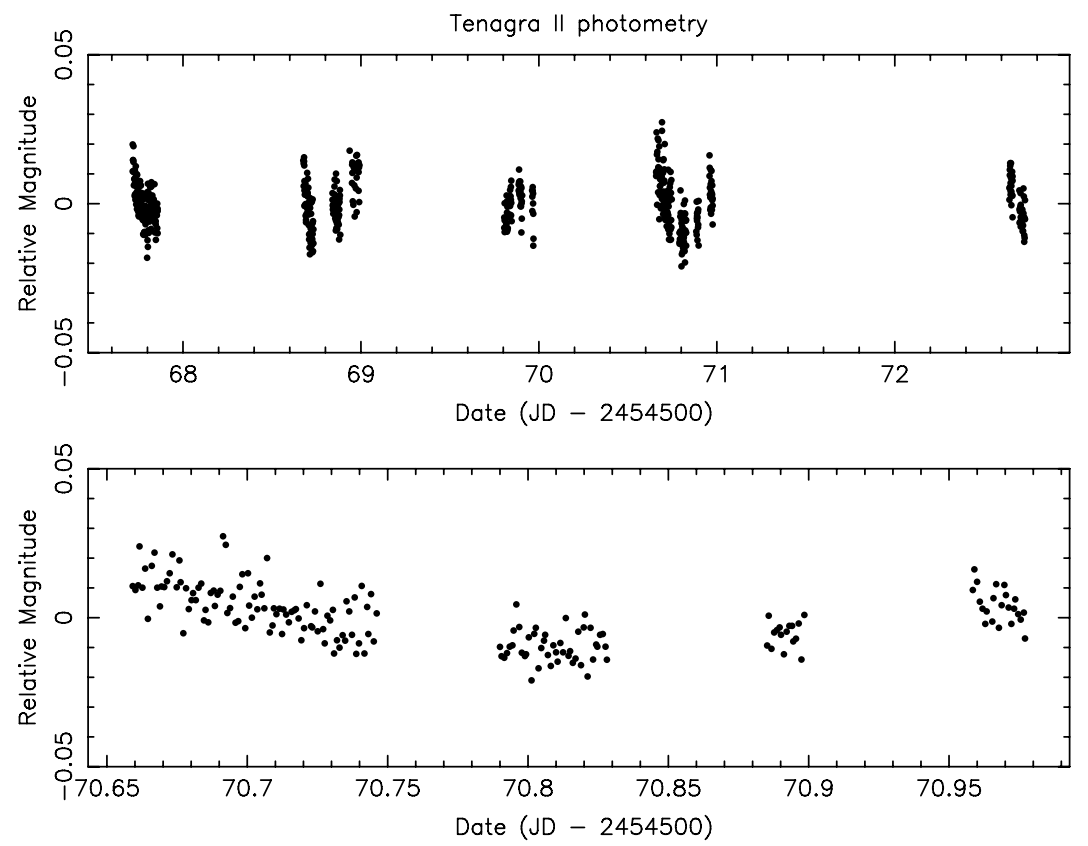

Figure 3. Photometry of TYC 2534-698-1 obtained using the Tenagra II 32 inch telescope.

5.7 millimags. No transit signature was detected on any of the nights observed.

Figure 3 presents the photometry from the Tenagra II telescope, covering a period of $\sim 6$ days shown in the top panel, and from a single night shown in the bottom panel. The relative magnitudes were computed using a single comparison star due to the limited field of view. These data also do not detect any transit event, but confirm the photometrically stable nature of the star. In this case, the standard deviation is 7.9 millimags, with residual airmass effects slightly increasing the nightly scatter.

\section{DATA ANALYSIS}

In this section, we present the results of analyzing the spectra, radial velocity, and photometric data, deriving the stellar parameters, best-fit orbital solution, mass, and transit ephemeris.

\subsection{Stellar Parameters and Mass Estimate}

While TYC 2534-698-1 has no Hipparcos parallax estimate, it does have Tycho-2 photometry $\left(B_{T}, V_{T}\right)$ as well as 2MASS photometry $(J, H, K)$. Tycho-2 photometry is known to degrade beyond $V_{T}>11.0$, but our target is bright enough that the photometric errors are not too large. Table 2 lists the photometric magnitudes and associated errors in the five different filters considered. To derive an approximate stellar temperature $\left(T_{\text {eff }}\right)$ we fit a series of synthetic SEDs to the photometric data using the CHORIZOS code (Maíz-Apellániz 2004). This yields a best-fit temperature of $5700 \mathrm{~K}$.

A more precise estimation of the spectral parameters was then performed using the stellar template obtained as part of the radial velocity observing program. The template was taken without the iodine cell in the beam path and allows the use 

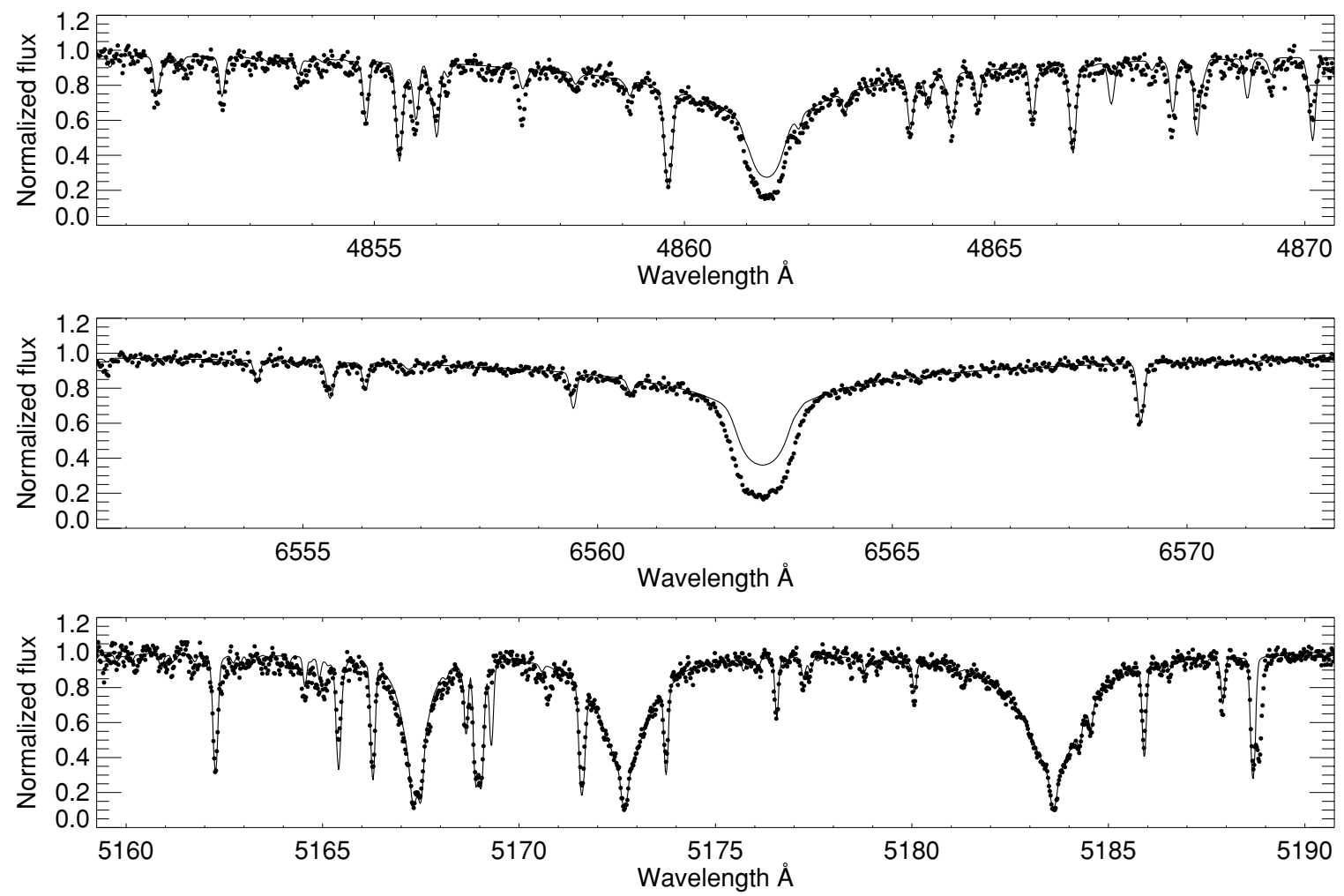

Figure 4. Continuum normalized plot of the high-resolution spectrum obtained with the HRS instrument. Wavelength regions shown are centered on $\mathrm{H} \alpha$, $\mathrm{H} \beta$, and $\mathrm{Mg}$ b. The best-fit stellar model (see Table 3) is plotted as a solid line.

Table 2

Tycho-2 \& 2MASS Photometry for TYC 2534-698-1

\begin{tabular}{lrc}
\hline \hline Filter & \multicolumn{1}{c}{ Magnitude } & Source \\
\hline$B_{T}$ & $11.567 \pm 0.061$ & Tycho- 2 \\
$V_{T}$ & $10.779 \pm 0.051$ & Tycho- \\
$J$ & $9.501 \pm 0.021$ & 2MASS \\
$H$ & $9.226 \pm 0.022$ & 2MASS \\
$K$ & $9.127 \pm 0.019$ & 2MASS \\
\hline
\end{tabular}

Table 3

Stellar Parameters

\begin{tabular}{lc}
\hline \hline Parameter & Value \\
\hline$T_{\text {eff }}$ & $5700 \pm 80 \mathrm{~K}$ \\
$\log g$ & $4.5 \pm 0.1$ \\
$\xi_{\mathrm{t}}$ & $1.6 \mathrm{~km} \mathrm{~s}^{-1}$ \\
$M_{\star}$ & $0.998 \pm 0.040 M_{\odot}$ \\
{$[\mathrm{Fe} / \mathrm{H}]$} & $-0.25 \pm 0.06$ \\
\hline
\end{tabular}

of the entire spectral range covered by the HRS. We use the latest MARCS model atmospheres (Gustafsson et al. 2008) for the analysis. Generation of synthetic spectra and the line analysis were performed using the turbospectrum code (Alvarez \& Plez 1998), which employs line broadening according to the prescription of Barklem \& O'Mara (1998). The line lists used are drawn from a variety of sources. Updated atomic lines are taken mainly from the VALD database (Kupka et al. 1999). The molecular species $\mathrm{CH}, \mathrm{CN}, \mathrm{OH}, \mathrm{CaH}$, and $\mathrm{TiO}$ are provided by B. Plez (see Plez \& Cohen 2005), while the NH, $\mathrm{MgH}$, and $\mathrm{C}_{2}$ molecules are from the Kurucz linelists. ${ }^{6}$ The solar abundances used here are the same as Asplund (2005). We use FeI excitation equilibrium and derived an effective temperature $T_{\text {eff }}=5700 \pm 80 \mathrm{~K}$ which is consistent with the effective temperature derived from photometry. We also fit the $\mathrm{H} \alpha$ and $\mathrm{H} \beta$ line wings, and derived $T_{\text {eff }}=5700 \pm 100 \mathrm{~K}$, which is also consistent with the $T_{\text {eff }}$ derived from photometry and SED. We also find $\log g=4.5 \pm 0.1$, based on ionization equilibrium of FeI and FeII lines and by fitting the wings of the Mgb lines. A microturbulence value $\xi_{\mathrm{t}}=1.6 \mathrm{~km} \mathrm{~s}^{-1}$ is

\footnotetext{
6 http://kurucz.harvard.edu/LINELISTS/LINESMOL/
}

derived by forcing weak and strong FeI lines to give the same abundances. We only used the FeI lines weaker the $120 \mathrm{~m} \AA$ in the analysis. These parameters are consistent with a G2V spectral type with a relatively low metallicity. To estimate the mass of the star we used Padova isochrones for metallicities of $Z=0.008,0.019,0.030$ and ages between 1.778 and 7.943 Gyr. Taking into account the uncertainties in $T_{\text {eff }}$ and $\log g$, from these isochrones we derive a mass estimate of $0.998 \pm 0.040 M_{\odot}$. Figure 4 shows the observed high-resolution spectrum, as well as the best-fit model, for wavelength regions centered on $\mathrm{H} \alpha, \mathrm{H} \beta$, and $\mathrm{Mg}$ b. Table 3 lists the derived stellar parameters.

\subsection{Model Fitting}

The radial velocity data were fit using the iterative gridsearch approach described by Kane et al. (2007). Fortunately, the allocated HET time allowed us to obtain complete phase coverage of the orbit, thus removing ambiguity at longer periods. As more measurements were obtained, the eccentric nature of the orbit quickly became clear, which necessitated even greater phase coverage in order to constrain the shape of the radial velocity variation. 

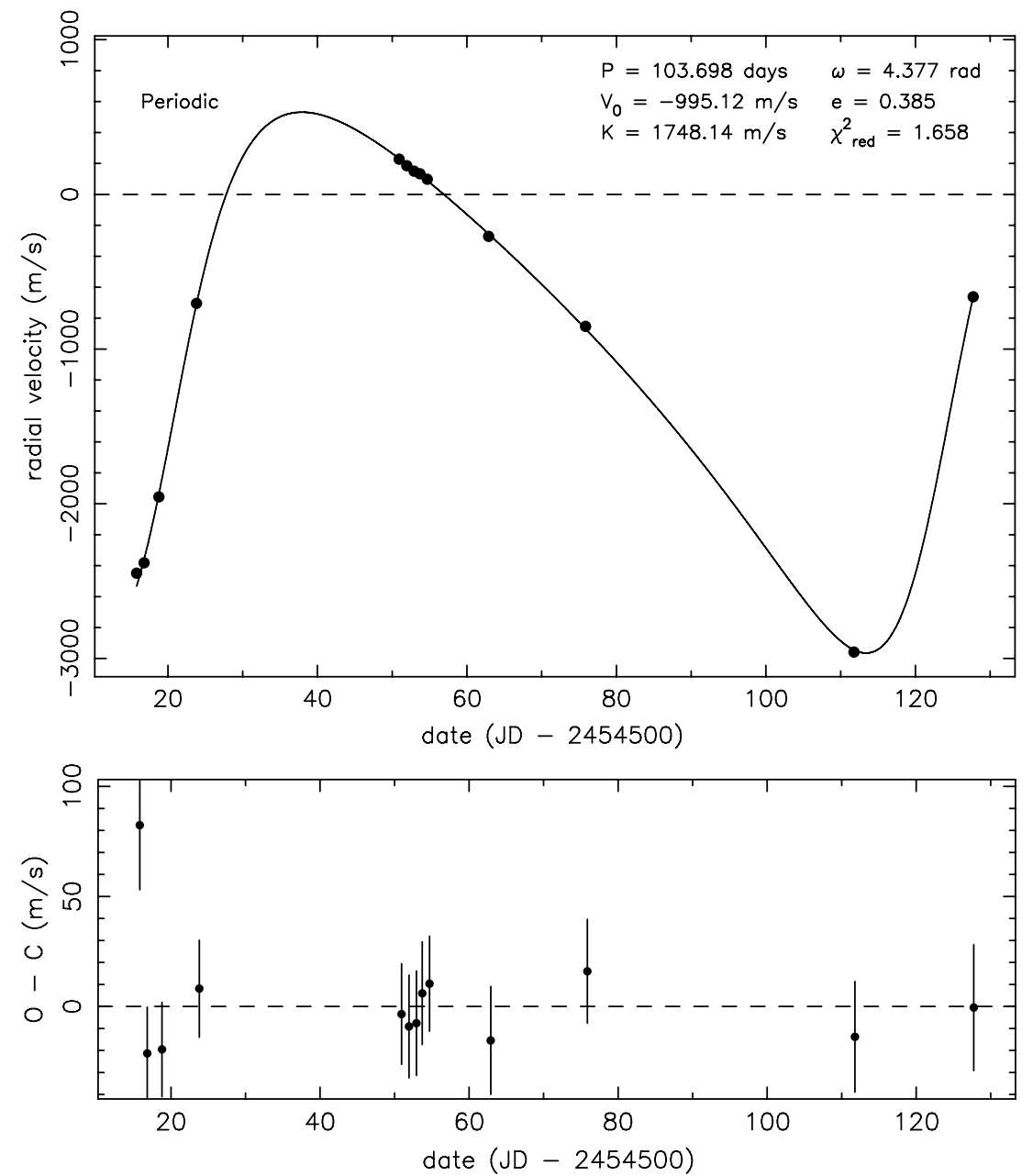

Figure 5. Radial velocity measurements of TYC 2534-698-1 along with the best-fit orbital solution (solid line). Error bars are shown but are small compared with the radial velocity amplitude in this case. The lower panel shows the residuals of the fit, observed minus calculated $(O-C)$.

Table 4

The Best-Fit Orbital Parameters

\begin{tabular}{lc}
\hline \hline Parameter & Value \\
\hline$P$ & $103.698 \pm 0.111$ days \\
$V_{0}$ & $-995.12 \pm 18.03 \mathrm{~m} \mathrm{~s}^{-1}$ \\
$K$ & $1748.14 \pm 27.48 \mathrm{~m} \mathrm{~s}^{-1}$ \\
$\omega$ & $4.377 \pm 0.013 \mathrm{rad}$ \\
$e$ & $0.385 \pm 0.011$ \\
$t_{0}$ & JD $2454519.389 \pm 0.137$
\end{tabular}

Notes. Includes period $P$, systemic velocity $V_{0}$, semiamplitude $K$, argument of periastron $\omega$, eccentricity $e$, and time at periastron $t_{0}$.

Shown in Figure 5 are the complete set of radial velocity measurements obtained at the HET as part of this program. Overplotted is a solid line which indicates the best-fit orbital model to the data, with a reduced $\chi^{2}$ of 1.658 . The lower panel shows the residuals from fitting the data with this model. The orbital parameters that provide the best-fit solution are shown in Table 4. The higher than expected eccentricity caused us to extend observations of this target until we were about to observe the turn around, which occurred at around JD 2454613. Using simulated data points, we calculated the optimal times of observations for the final few measurements which would yield the greatest constraints on the orbital period.
Given the phase coverage and eccentricity for this system, it is important to determine the reliability of the fitted parameters. By producing $\chi^{2}$ maps of parameter space, one can see where the local and global minima lie in relation to the fit values for those parameters. This is achieved by varying one parameter and holding all of the others fixed. Figure 6 shows the $\chi^{2}$ maps for the period, semiamplitude, eccentricity, and argument of periastron. The phase coverage of the orbit produces significant global minima which match the best-fit values shown in Table 4 . The acquisition of the last two radial velocity measurements in particular allowed us to constrain the value of the period.

\subsection{Companion Inclination and Mass Estimate}

The mass function can be related to the observed period, eccentricity, and radial velocity semiamplitude as

$$
\frac{(m \sin i)^{3}}{\left(M_{\star}+m\right)^{2}}=\frac{P\left(1-e^{2}\right)^{\frac{3}{2}} K^{3}}{2 \pi G},
$$

where $M_{\star}$ is the mass of the primary and $m$ is the mass of the secondary. Without having observed the transit we cannot constrain $\sin i$ directly, leading to the degeneracy of the companion mass with assumed inclination angle. Using the derived stellar mass $\left(0.998 \pm 0.040 M_{\odot}\right)$ for the primary with the orbital parameters determined from the radial velocity model (Table 4) we determine that the minimum mass of the companion 

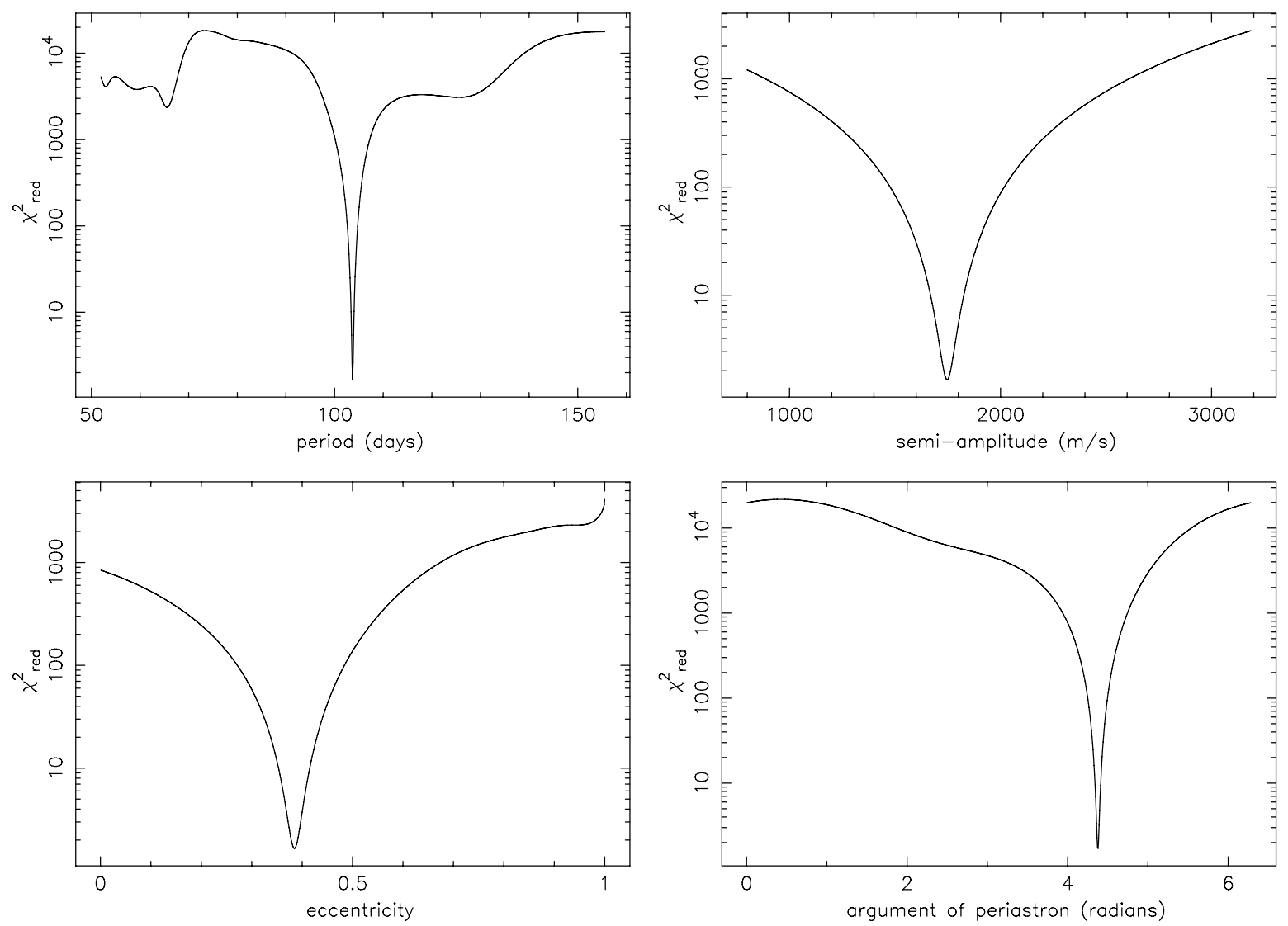

Figure 6. $\chi^{2}$ maps of parameter space showing the locations of the local and global minima for the period, semiamplitude, eccentricity, and argument of periastron.

for an edge-on orbit $(\sin i=1)$ is $0.0373 \pm 0.011 M_{\odot}$. Using an upper-mass limit of $0.08 M_{\odot}$ for a brown dwarf, Equation (1) predicts that a companion of this mass would have an orbital inclination of $i=28.5$ to reproduce the radial velocity curve observed.

For a chance orientation of the orbital inclination, the probability that the inclination angle $i$ is less than an angle $\theta$ is given by

$$
p(i<\theta)=1-\cos \theta .
$$

The probability of the companion having an inclination less than 28.5 , and therefore being more massive than a brown dwarf, is only $12.1 \%$. Figure 7 shows the range of possible masses for the companion as a function of the inclination angle. Masses of the companion close to that of the primary (for very low inclination angles) are excluded based on the absence of any secondary spectra in echelle data. However, the constraint is rather weak since the $\mathrm{S} / \mathrm{N}$ of the spectra itself is not very high, making it difficult to exclude fainter $\mathrm{M}$ star companions.

\subsection{Transit Ephemeris}

The possible transit of the stellar companion motivated a considerable amount of the photometric campaign that was undertaken (see Section 2.2). As the knowledge regarding the orbital parameters evolved, new transit ephemerides were calculated and transit windows were communicated to the observers. The time of predicted transit based upon the complete orbital solution is shown in Figure 8. The solid vertical lines correspond to nights on which photometric data were acquired. Unfortunately, the final radial velocity measurements obtained shifted the transit window such that photometry was not obtained on the night

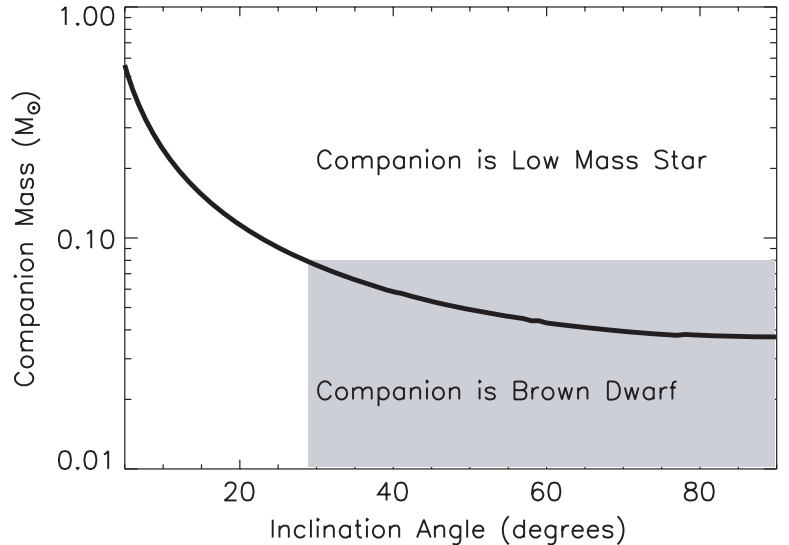

Figure 7. This figure shows the mass of the companion for different values of the inclination angle $(i)$. The mass of the companion falls in the brown dwarf regime for all inclination angles larger than 28.5 .

of predicted transit. In addition, the final orbital solution was checked with the original SuperWASP observations and did not match any of the observed transits. Although six transits were observed by SuperWASP, they were only observed in one camera and were not detected by the other cameras looking at the same field. The photometry presented in Section 2.2 is more than adequate, both in coverage and precision, to detect the $\mathrm{Su}$ perWASP transit if it is real. Thus the SuperWASP detection is likely to be spurious in nature.

The orbital orientation of this system is such that the periastron location is behind the star. As such, the probability of a transit occurring is $0.89 \%$, but the probability of a secondary 


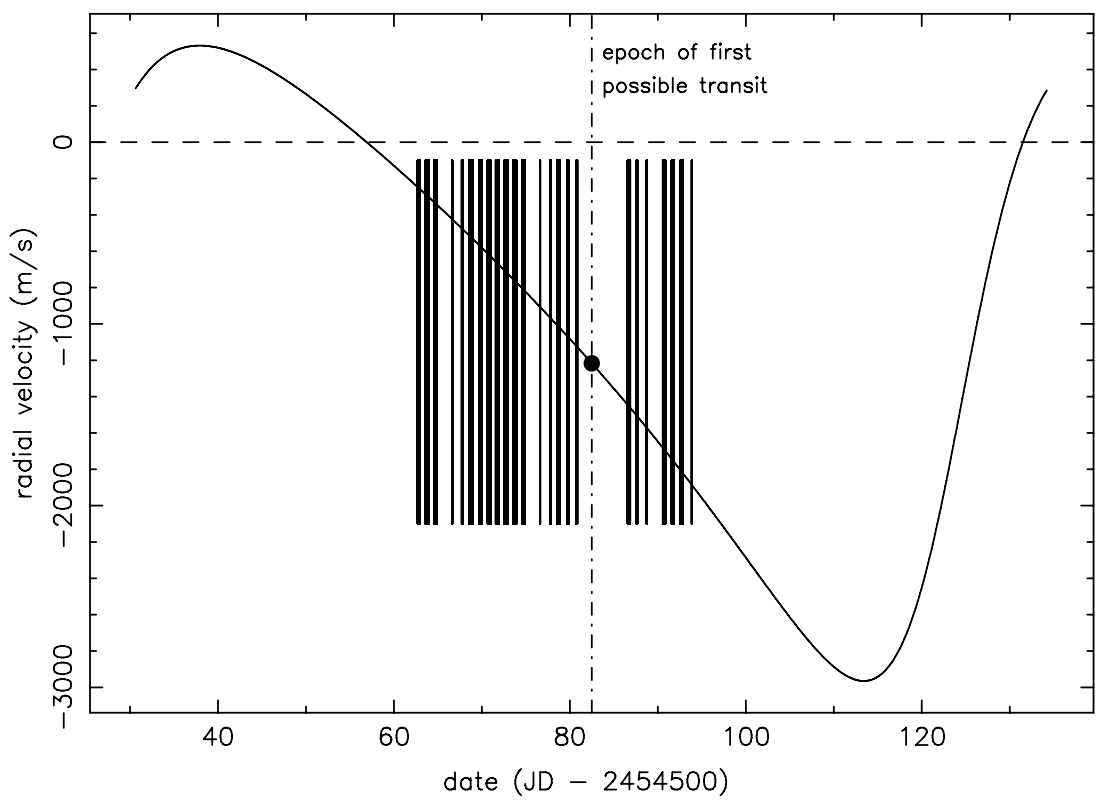

Figure 8. Predicted transit time for the stellar companion. The vertical solid lines indicate the dates of photometric coverage of the target relative to the transit window.

eclipse is $1.89 \%$. These are small odds indeed, but the possibility of constraining the inclination and therefore the mass of the companion make this a compelling target to study further. The duration of a planetary transit for a circular orbit is generally given by

$$
t_{\text {circ }}=\frac{P}{\pi} \arcsin \left(\frac{\sqrt{\left(R_{p}+R_{\star}\right)^{2}-(a \cos i)^{2}}}{a}\right),
$$

where $R_{p}$ and $R_{\star}$ are the radii of the planet and parent star respectively. For our target, and assuming a companion radius of one Jupiter radius, this amounts to a $9.38 \mathrm{hr}$ duration. However, the eccentricity of the companion changes this duration significantly. Using the transit duration scaling factor for eccentric orbits provided by Burke (2008),

$$
\frac{t_{\mathrm{ecc}}}{t_{\mathrm{circ}}}=\frac{\sqrt{\left(1-e^{2}\right)}}{(1+e \cos (\omega-\pi / 2))},
$$

we compute a transit duration of $13.61 \mathrm{hr}$ and a secondary eclipse duration of $6.35 \mathrm{hr}$. If we assume a radius of 1 Jupiter radius, the predicted transit depth is $1 \%$. If we assume a radius of 2 Jupiter radii, the predicted transit depth becomes $4.2 \%$. Following up the target at the predicted transit times is therefore a feasible task with high scientific yield.

\section{DISCUSSION}

Based on random inclinations the probability of the companion being a brown dwarf is high. However, given that low-mass stars in binary systems are more frequent than brown dwarfs at these separations, it is necessary to constrain the mass of the companion and exclude the possibility that it may be a low-mass star. In this section, we explore the various techniques possible to accomplish this and their relevance and applicability for TYC 2534-698-1.

Astrometric Orbit: Using the orbital parameters, we can estimate the astrometric perturbation expected for different values of the companion mass. The orbital parameters yield a semimajor axis of $0.4417 \mathrm{AU}$ for the orbit and the best-fit spectroscopic stellar model indicates that the absolute magnitude $M_{V}$ is 4.77, yielding an approximate distance estimate of $\sim 155$ parsecs. For the case of the minimum mass companion, the astrometric perturbation is only $100 \mu$ arcsec, and is $220 \mu \operatorname{arcsec}$ if the companion were $0.08 M_{\odot}$. The small semi-major axis coupled with large distance to the star makes astrometric detection difficult. The Hubble Space Telescope fine guidance sensors are capable of sub-milliarcsecond astrometry, but a 1 milliarcsecond signature only appears when the companion mass exceeds $0.36 M_{\odot}$. Constraining the astrometric signature to demonstrate that the object is indeed a brown dwarf will require the astrometric accuracy of planned instruments like Space Interferometry Mission (SIM).

Transit and Secondary Eclipse: The detection of a transit would constrain the inclination of the companion orbit and unambiguously confirm its mass to be in the brown dwarf regime. The long-period eccentric orbit however leads to a low probability of only $\sim 0.9 \%$ for the transit. The predicted transit duration of $13.6 \mathrm{hr}$ is also too long for the transit to be observed with most available telescopes. Longer-period transits are also more difficult to detect from the ground due to correlated red noise and other systematics. The observed eccentricity and orientation of the orbit makes the detection of a secondary eclipse more likely than that of a transit. The probability of this is $\sim 1.9 \%$ which, while still small, is a factor of 2 larger than the probability of a primary transit. The secondary eclipse, if present, would be easier to detect with Spitzer than similar transits of planets due to the self-luminous nature of the brown dwarf candidate.

Constraints from Radial Velocity: High-precision radial velocity observations can themselves be used to exclude certain mass ranges of the companion. If an iodine cell were used then the presence of a brown dwarf companion spectra would manifest itself as increased noise in the radial velocity data when the different spectral chunks are being compared. Such a technique has been used by Kürster et al. (2008) to detect a probable brown dwarf around GJ 1046. Meaningful constraints with this technique however require very high-precision velocities at $2-3 \mathrm{~ms}^{-1}$. Even with such a precision it is not possible to distinguish between a brown dwarf and a very low-mass star. The 
primary is also relatively faint, making the acquisition of precision radial velocities very time consuming.

Interferometric Observations: The projected maximum separation of the companion from the primary is only $\sim 2$ mas, making any direct detection difficult. The projected separation is smaller than the 5 mas resolution (at $2.2 \mu \mathrm{m}$ ) of even the $85 \mathrm{~m}$ Keck interferometer baseline, and the contrast requirements too high unless the companion were an early $\mathrm{M}$ dwarf at a high inclination.

\section{CONCLUSIONS}

We have reported the discovery of a low mass candidate around TYC 2534-698-1. Confirmation of a mass less than $0.08 M_{\odot}$, or the detection of a transit, would make this one of the very few known brown dwarfs in the "desert." While the detection of a transit is a low probability, such a detection would make this the only bright transiting brown dwarf. The long transit timescale, while disadvantageous for the detection, is actually a tremendous advantage for transmission spectroscopy (if a transit is detected) to probe the atmosphere of the candidate (Redfield et al. 2008). In the event that transits do not occur, tighter limits on the mass of the companion will have to await future missions such as SIM, which can easily detect the small predicted amplitude of the astrometric signal.

The authors would like to thank Leslie Hebb for several useful discussions, the HET staff astronomers for performing the observations, and Bertrand Plez for providing the new version of the turbospectrum code which uses the latest MARCS models. We would also like to thank University of Texas at Austin for awarding Director's Discretionary Time which allowed us to obtain complete phase coverage of the target. The HET is a joint project of the University of Texas at Austin, the Pennsylvania State University, Stanford University, Ludwig-MaximiliansUniversität München, and Georg-August-Universität Göttingen. The HET is named in honor of its principal benefactors, William P. Hobby and Robert E. Eberly.

\section{REFERENCES}

Alvarez, R., \& Plez, B. 1998, A\&A, 330, 1109

Asplund, M. 2005, ARA\&A, 43, 481

Barklem, P. S., \& O’Mara, B.J. 1998, MNRAS, 300, 863

Burke, C. J. 2008, ApJ, 679, 1566

Chabrier, G., \& Baraffe, I. 2000, ARA\&A, 38, 337

Cochran, W. D., et al. 2004, ApJ, 611, L133

Deleuil, M., et al. 2008, A\&A, 491, 889

Gustafsson, B., Edvardsson, B., Eriksson, K., Jørgensen, U. G., Nordlund, Å., \& Plez, B. 2008, A\&A, 486, 951

Ireland, M. J., Kraus, A., Martinache, F., Lloyd, J. P., \& Tuthill, P. G. 2008, ApJ, 678,463

Johns-Krull, C. M., et al. 2008, ApJ, 677, 657

Kane, S. R., et al. 2008, MNRAS, 384, 1097

Kane, S. R., Schneider, D. P., \& Ge, J. 2007, MNRAS, 377, 1610

Kürster, M., Endl, M., \& Reffert, S. 2008, A\&A, 483, 869

Kupka, F., Piskunov, N., Ryabchikova, T. A., Stempels, H. C., \& Weiss, W. W. 1999, A\&AS, 138, 119

Maíz-Apellániz, J. 2004, PASP, 116, 859

Marcy, G. W., \& Butler, R. P. 2000, PASP, 112, 137

Metchev, S., \& Hillenbrand, L. 2009, ApJ, in press (arXiv:0808.2982)

Perryman, M. A. C., et al. 1997, A\&A, 323, L49

Plez, B., \& Cohen, J. G. 2005, A\&A, 434, 1117

Redfield, S., Endl, M., Cochran, W. D., \& Koesterke, L. 2008, ApJ, 673, L87

Reffert, S., \& Quirrenbach, A. 2006, A\&A, 449, 699

Stassun, K. G., Mathieu, R. D., \& Valenti, J. A. 2006, Nature, 440, 311

Tull, R. G. 1998, Proc. SPIE, 3355, 387

Zucker, S., \& Mazeh, T. 2000, ApJ, 531, L67

Zapatero Osorio, M. R., Lane, B. F., Pavlenko, Y., Martín, E. L., Britton, M., \& Kulkarni, S. R. 2004, ApJ, 615, 958 